\title{
Mesotelioma en paciente colombiano con sobrevida mayor a tres años: presentación de caso
}

\author{
Mesothelioma in a Colombian Patient \\ over three years: Case Presentation \\ Mesotelioma em paciente colombiano \\ com sobrevivência superior a três anos: \\ apresentação de caso
}

\author{
Juanita Betancourt1* \\ Juan C. Hernandez, PhD ${ }^{1}$ \\ Carlos Alberto Rodríguez, MD, Esp. CTO² \\ Juan Carlos Quintana-Castillo, Bact. PhD ${ }^{3}$ \\ Carlos Augusto Rojas, MD, Esp. Anat. Pat. ${ }^{3}$
}

Recibido: 17 de marzo de $2020 \cdot$ Aceptado: 6 de agosto de 2020

Doi: https://doi.org/10.12804/revistas.urosario.edu.co/revsalud/a.10154

Para citar este artículo: Betancourt J, Hernández JC, Rodríguez CA, Quintana-Castillo, JC, Rojas, CA. Mesotelioma en paciente colombiano con sobrevida mayor a tres años: presentación de caso. Rev Cienc Salud. 2021;19(1):1-10. https://doi.org/10.12804/revistas. urosario.edu.co/revsalud/a.10154

\section{Resumen}

Introducción: el mesotelioma epitelioide es un tumor que se desarrolla en las capas embrionarias mesoteliales; es de etiología desconocida, pero se relaciona con la exposición al asbesto, con una presentación clínica inespecífica y con un pronóstico de sobrevida corto después del diagnóstico. Presentación del caso: hombre de profesión mecánico automotor, con tos sin expectoración, disnea, hipertermia y emaciación

1 Facultad de Medicina, Universidad Cooperativa de Colombia, sede Medellín (Colombia).

2 Facultad de Medicina, Universidad Militar Nueva Granada (Colombia).

3 Facultad de Medicina, Universidad Cooperativa de Colombia, sede Medellín, y Facultad de Medicina, Universidad de Antioquia (Colombia).

Juanita Betancourt: oRCID https://orcid.org/0000-0003-1219-6568

Juan C. Hernandez: oRCID https://orcid.org/0000-0002-9200-5698

Juan Carlos Quintana: orciD https://orcid.org/0000-0002-7923-9158

Carlos Alberto Rodríguez: ORCID https://orcid.org/0000-0003-0129-8438

Carlos Augusto Rojas: oRCID https://orcid.org/0000-0002-3184-7875

Autora de correspondencia: juanita.betancourt@campusucc.edu.co o betancourtj06@gmail.com 
posterior a la extracción quirúrgica de lipomas que afectaban el tórax, quien posteriormente fue diagnosticado con mesotelioma epitelioide maligno con ubicación en la pleura del hemitórax derecho y fue tratado con toracotomía, quimioterapia con los medicamentos pemetrexed y cisplatino y sesiones de radioterapia, que mostraron un aumento en la sobrevida 3 tres años. Conclusión: este caso permitió identificar que el uso de la pleurodesis química con quimioterapia como tratamiento podría ser responsable del aumento de la esperanza de vida y la calidad de esta en los pacientes que padecen este tipo de tumor.

Palabras clave: mesotelioma; asbestosis; mesodermo; cisplatino; pemetrexed.

\section{Abstract}

Introduction: Epithelioid mesothelioma is a tumor that develops in the mesothelial embryonic layers; it is of an unknown etiology, but it is related to asbestos exposure with a nonspecific clinical presentation and a short survival prognosis after diagnosis. Case presentation: An automotive mechanic patient presents with cough without expectoration, dyspnea, hyperthermia, and emaciation following surgical removal of lipomas. This affected the chest and the patient was subsequently diagnosed with malignant epithelioid mesothelioma located in the pleura of the right hemithorax. The patient was treated with thoracotomy, chemotherapy with the drugs pemetrexed and cisplatin, and radiation therapy sessions which resulted in an increased survival rate at 4 years. Conclusion: This case report identifies the use of chemical pleurodesis in combination with chemotherapy as an effective treatment for increasing the life expectancy and quality of life in patients suffering from this type of tumor.

Keywords: Mesothelioma; asbestosis; mesoderm; cisplatin; pemetrexed.

\section{Resumo}

Introdução: o mesotelioma epitelióide é um tumor que se desenvolve nas camadas embrionárias mesoteliais; é de causa desconhecida, mas está relacionado com a exposição ao amianto e possui uma manifestação clínica inespecífica e com prognóstico de sobrevivência curto após o diagnóstico. Apresentação do caso: o paciente é um mecânico automotivo, que apresentou tosse seca, dispneia, hipertermia e emagrecimento posterior a extração cirúrgica de lipomas que afetavam o tórax sendo posteriormente diagnosticado com mesotelioma epitelióide maligno localizado na pleura do hemitórax direito e foi tratado com toracotomia, quimioterapia com os medicamentos pemetrexed e cisplatino além de sessões de radioterapia, mostrando um aumento de expectativa de vida para 4 anos. Conclusão: este estudo de caso permite identificar que o uso da pleurodese química com quimioterapia como tratamento poderia ser a responsável pelo aumento da expectativa e qualidade de vida em pacientes acometidos por este tipo de tumor.

Palavras-chave: mesotelioma; asbestose; mesoderma; cisplatino; pemetrexed.

\section{Introducción}

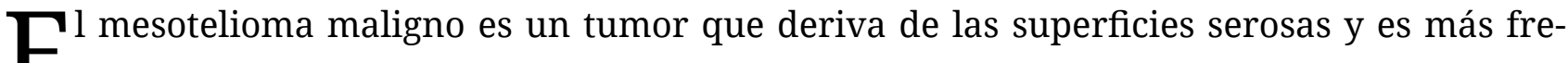
Ecuente en la cavidad pleural que en el peritoneo, que es derivado de las células mesoteliales que se encuentran en pulmones, peritoneo, pericardio y testículos (1). La incidencia del mesotelioma está en aumento; incluso es más común que los cánceres de vejiga y huesos. Con frecuencia, los pacientes evidencian una sintomatología de disnea, angina, tos y emaciación. El tumor puede invadir la pleura visceral y parietal y se extiende a estructuras 
adyacentes, por lo que es de mal pronóstico, al punto que los pacientes tienen una mediana de supervivencia de seis a dieciocho meses después del diagnóstico (2).

Para el diagnóstico de esta enfermedad se realiza un estudio inmunohistoquímico en el cual se busca clasificar las células en los diferentes tipos, como son epitelioide, sarcomatoso y mixto. Adicionalmente, un estudio de imágenes radiológicas apoya el diagnóstico de derrame pleural (3).

El tratamiento actual incluye cirugía (pleurectomía o neumonectomía extrapleural), seguida de radioterapia, aunque recientemente se ha añadido el uso de quimioterapia con medicamentos como el pemetrexed, cisplatino o carboplatino. El tratamiento paliativo contribuye a mejorar la calidad de vida y a alcanzar un buen control sintomático durante años tras el diagnóstico (4).

\section{Presentación del caso}

$\Gamma^{l}$ caso corresponde a un hombre de 56 años de edad, residente en Ibagué (Tolima, Colombia), Ede profesión mecánico automotor/industrial, a quien en enero de 2014 se le hallaron tres masas en región torácica con diagnóstico inicial de lipomas, y a quien se remitió a cirugía con el fin de retirárselos. Se le solicitaron exámenes preoperatorios, entre estos una radiografía de tórax (figura 1). Los lipomas fueron extraídos por cirugía ambulatoria y se tomaron muestras de tejido muscular de las regiones torácica y pleural que se remitieron a estudio histopatológico en la ciudad de Bogotá.

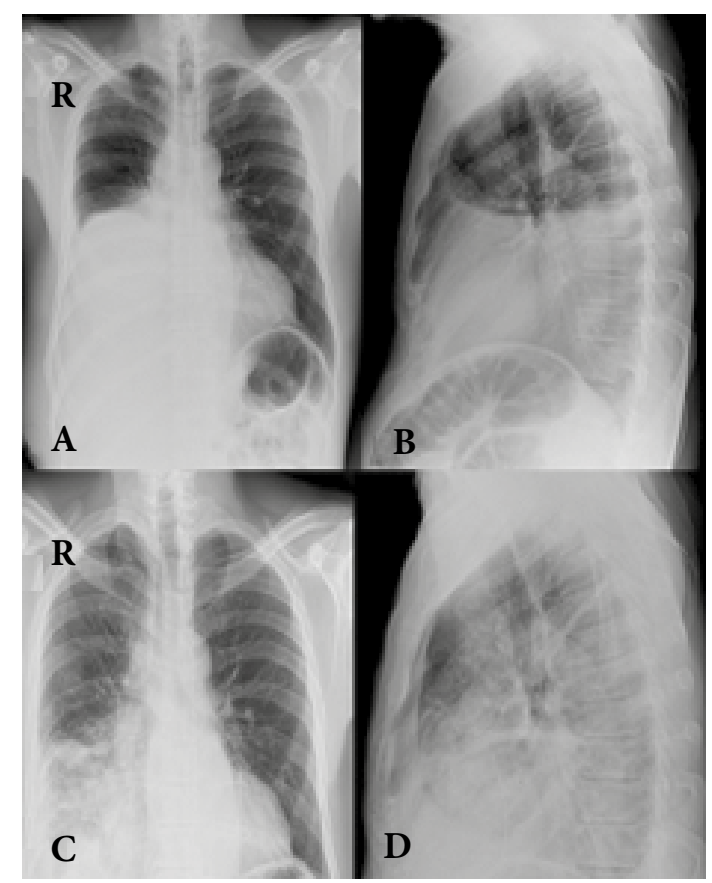

Figura 1. Radiografía de tórax: en la proyección posterior anterior se evidencia opacidad en el tercio interior del hemitórax izquierdo con borramiento del hemidiafragma con ascenso pulmonar ipsilateral (A). En la proyección lateral (B) se visualiza opacidad en 2/3 del hemitórax. En (C) se observa aumento de la silueta pulmonar y del diámetro pulmonar con presencia de nódulos y posible afectación de los tejidos adyacentes. (D) Hay expansión pulmonar con presencia de consolidación aparentemente paraneumónica 
Trascurridos tres meses desde la intervención, el paciente evidenciaba una tos persistente con disnea continua; adicionalmente, emaciación, fatiga, diaforesis nocturna, astenia y adinamia. El paciente ingresó a urgencias, y en el examen físico se auscultó líquido en la región torácica derecha, confirmado por una radiografía, donde se evidenciaba colapso pulmonar izquierdo (figuras 2a y 2b). El paciente fue hospitalizado con un peso de $49 \mathrm{~kg}$ y fiebre de $38^{\circ} \mathrm{C}$ y se le solicitaron estudios de laboratorio. Según estos, los resultados fueron: hematocrito al $41.5 \%$; la hemoglobina: $13.8 \mathrm{~g} / \mathrm{dL}$; neutrófilos: $13.8 \mathrm{~g} / \mathrm{dL}$, y nitrógeno ureico sanguíneo: $19 \mathrm{mg} / \mathrm{dL}$, para orientar el diagnóstico. Como medida clínico-quirúrgica se le drenó el líquido localizado en la región torácica, que dio como resultado un descenso del colapso pulmonar (figuras 2c y 2d). En el manejo hospitalario el paciente fue remitido a la ciudad de Bogotá, en junio de 2014, a una institución hospitalaria de mayor complejidad de atención en salud.

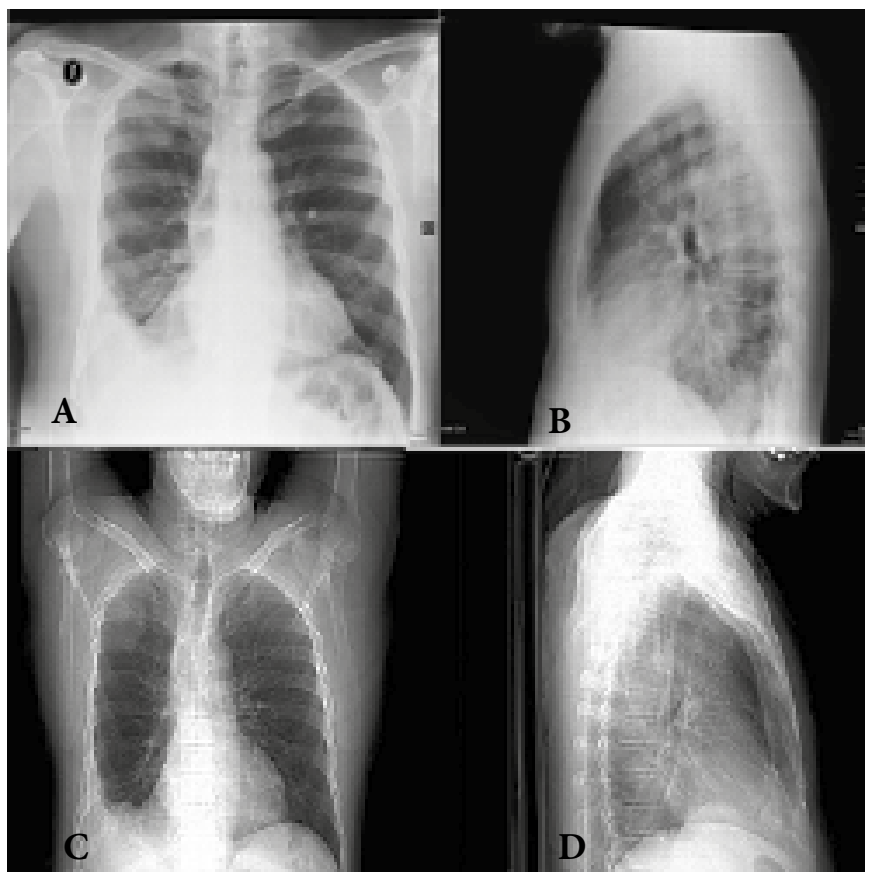

Figura 2. Radiografía de tórax (A y B). Se observa engrosamiento pleural con consolidación unilateral en el ángulo costo-diafragmático y desplazamiento mediastinal, con visualización de la rama bronquial aparentemente normal. (C y D) Se detalla un aumento del espacio intrapulmonar con evidencia de múltiples consolidaciones en el ápice pulmonar y en el ángulo costo-diafragmático derecho con desplazamiento del mediastino posterior. Pulmón izquierdo aparentemente normal.

De acuerdo con las pruebas inmunohistoquímicas, se diagnosticó mesotelioma epitelioide maligno, positivo a citoqueatina AE1/AE3, citoqueratinas 5 y 7, citoqueratina de alto peso molecular, calretinina D2-40 y antígeno epitelial de membrana (figuras 3a-3d). La medida quirúrgica es la realización de una toracotomía, con pleurodesis química y se dejó con dren de Blake hasta que se detuviera la producción de líquido pleural "anormal”. Se remitió a inicio de tratamiento oncológico. 


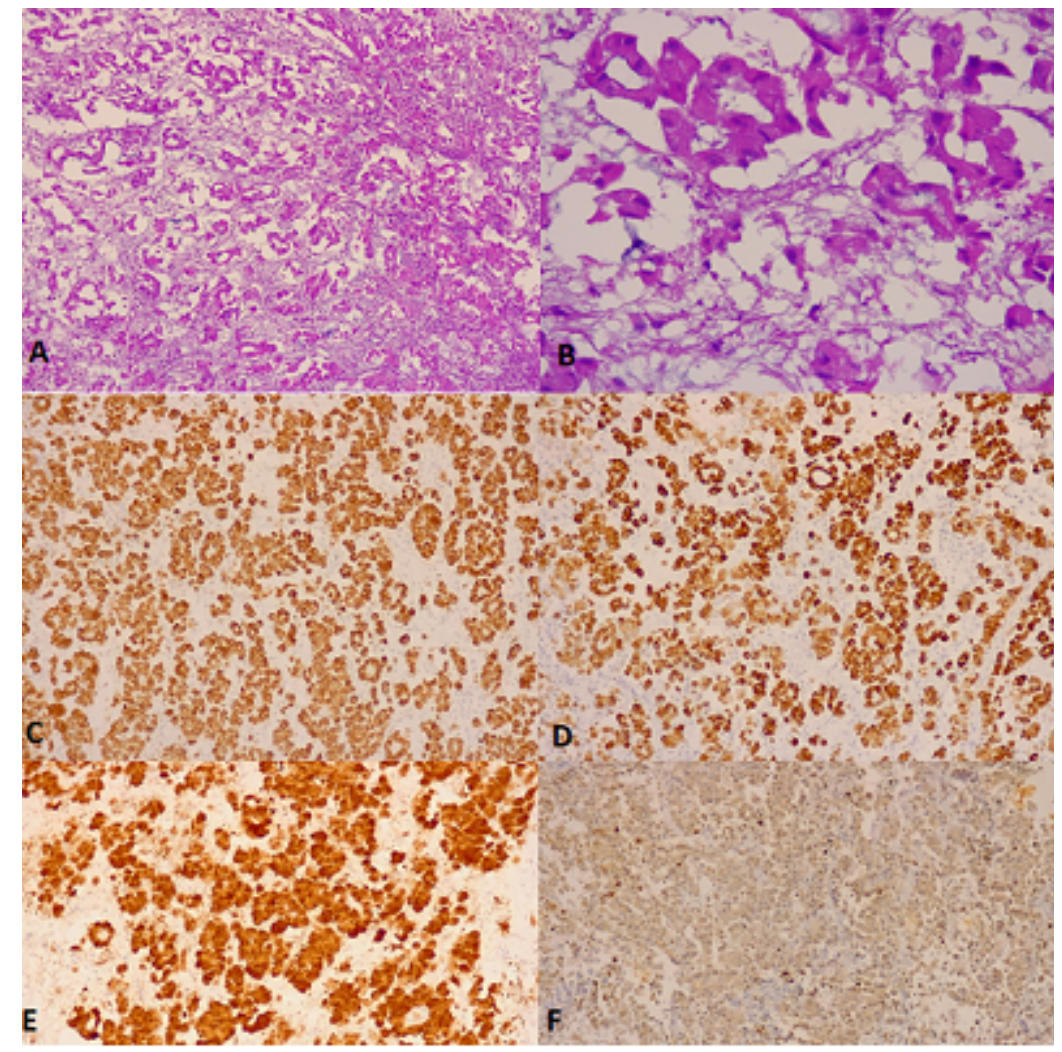

Figura 3. Los cortes con tinción de hematoxilina-eosina observados (A) 10X y (B) 40X muestran neoplasia infiltrativa en los tejidos blandos con presencia de focos de necrosis, estroma de aspecto desmoplásico, se compone de células poligonales de citoplasma eosinófilo amplio, con núcleos de tamaño variable visibles, que forman grupos similares a glándulas. Los marcadores de inmunohistoquímica son positivos para el cóctel de (C) 10X y (D) 10X citoqueratinas 5, 6 y 7. La positividad fuerte y difusa para la calretinina (E) 10X y (F) 4X en patrón membranosos para D240 y la negatividad para el antígeno carcinoembrionario policlonal son cosistentes con el diagnóstico de mesiotelioma epitelio de maligno.

En julio de 2014 inició quimioterapia con pemetrexed (inhibidor de enzimas dependientes de folatos, que bloquea la síntesis de nucleótidos) y cisplatino (inhibidor de la replicación de ADN y ARN). Luego fue dado de alta con indicación de oxígeno y el dren de Blake para evitar el colapso pulmonar. Este dren se le retiró cuatro meses después de su ubicación, puesto que el paciente dejó de producir líquido. En el entretanto continuó con éxito sus sesiones de quimioterapia, debido a las cuales se evidenció la desaparición de la fiebre y un aumento del peso (entre $3 \mathrm{y}$ $5 \mathrm{~kg}$ por mes).

Seis meses después, el paciente fue valorado por el cirujano, y los estudios imagenológicos evidenciaron que las múltiples masas de la región torácica y la parte posterior del corazón disminuyeron de tamaño, por lo que le sugirieron seis sesiones más de quimioterapia. Sin embargo, el paciente desarrolló alergia a uno de los medicamentos con prurito que comenzó en el tórax y que se diseminó a las extremidades, tratado con hidrocortisona. Las sesiones de quimioterapia se hicieron, entonces, más continuas para disminuir las masas y se consideró la opción de extraerlas quirúrgicamente. 
Para enero de 2016, el paciente tuvo una marcada disminución en el tamaño y número de las masas pulmonares, por lo que se siguió con el tratamiento. Se le solicitó una tomografía por emisión de positrones contrastado. El resultado del examen mostró un engrosamiento en las pleuras del hemitórax derecho, con derrame pleural escaso, aunque no se pudo medir el grosor del tumor por la falta del medio de contraste.

En junio de 2016, le cambiaron los medicamentos de quimioterapia por gemcitabina, debido a la persistencia de sintomatología alérgica, con doble refuerzo a los días 1 y 8 de tratamiento. Continuó con estos hasta mayo de 2017. En esa fecha, se pasó a medicamentos de segunda línea, puesto que el paciente reaccionó con alergia a uno de los medicamentos. El pemetrexed fue remplazado por vinorelbina con tres refuerzos a los días 1, 8 y 15. El paciente falleció en febrero de 2018.

\section{Discusión}

El mesotelioma es un tumor que deriva del mesotelio, una de las capas de células del desarrollo dará lugar a la pleura, al pericardio, al peritoneo y a la túnica albugínea del testículo. Este origen mesodérmico le confiere la potencialidad de desarrollar un componente epitelioide y otro sarcomatoso. El mesotelioma puede desarrollarse en las cavidades donde se desarrolla el mesotelio (5).

Desde la década de 1960 se conoce la asociación de este tumor con la exposición al asbesto $(6,7)$. Aproximadamente, en el $80 \%$ de los casos de mesotelioma hay una relación causa-efecto que se debe a la exposición laboral con el asbesto y donde múltiples profesiones se ven implicadas.

Una revisión publicada en 1984 informó que el talco con el que se realiza la pleurodesis química en los pacientes con mesotelioma es un silicato magnésico hidratado, cuya composición aproximada es de sílice, magnesio y agua. Se sugería que esta mezcla puede inducir apoptosis en células tumorales e inhibir la angiogénesis, con lo que se contribuye a un mejor control de la efusión pleural maligna, aunque la célula mesotelial pleural es el objetivo principal y desempeña un papel fundamental en todo el proceso, incluyendo inflamación difusa, desequilibrio pleural coagulación-fibrinólisis, reclutamiento de fibroblastos y la subsiguiente proliferación y producción de colágeno después de la aplicación intrapleural del agente esclerosante $(8,9)$.

Un artículo publicado por Alfonso Oropesa et al., en 2012, habló del tratamiento del derrame pleural paraneoplásico y demostró que la pleurectomía es un buen método para el control de derrame y una técnica quirúrgica con una efectividad del $99 \%$, pero a costa 
de una mortalidad de entre el 6\% y el 10\%, y una morbilidad del $23 \%$ (10). Por parte de la pleurodesis, parece tener menor morbilidad y mortalidad, menos efectos colaterales y previene la recurrencia del derrame.

En un ensayo clínico aleatorizado en 2008, realizado en el Reino Unido, con pacientes diagnosticados con mesotelioma y que recibieron ciclos cortos de quimioterapia, se observó que aquellos sometidos adicionalmente a neumonectomía extrapleural presentaban mayor supervivencia, comparados con los pacientes que no se sometieron a este procedimiento (nueve vs. siete meses, respectivamente) (11).

Bessel Suffian Al-Alao et al., en un artículo publicado en 2012, describieron las características asociadas a la supervivencia de los pacientes que padecen mesotelioma pleural maligno. Informaron que a los pacientes a quienes se les realiza decorticación y neumonectomía no les afecta la supervivencia; mientras que la quimioterapia se asocia con una mejor supervivencia, y la pleurodesis con talco es un determinante independiente para la supervivencia (12). Para este caso, la sobrevida del paciente fue más alta, posiblemente, mediada por la combinación de tratamientos.

Bielsa et al. describieron la importancia pronóstica del análisis del líquido pleural en pacientes con derrame maligno (13). Evidenciaron que la mediana de supervivencia global de los pacientes con derrame pleural maligno fue de cinco meses después del diagnóstico y que la supervivencia varió significativamente según el tipo de tumor primario: diecisiete meses para el mesotelioma, en este caso (14). Además, informaron que la predicción de supervivencia en pacientes que padecen mesotelioma pleural maligno a través de tomografía computarizada demostró que los cambios en el volumen del tumor después de dos ciclos de quimioterapia predijeron la supervivencia en pacientes con mesotelioma pleural maligno, la cual fue de trece meses más larga que aquellos pacientes con un volumen de tumor aumentado (14). Si bien la supervivencia del paciente en este caso fue mayor y no se medió el líquido pleural, su tratamiento y seguimiento evidenciaron su eficacia en el aumento de la supervivencia.

Weder et al. publicaron un ensayo multicéntrico donde informaron una mediana de supervivencia de 23 meses a quienes fueron sometidos a quimioterapia y neumonectomía extrapleural (15). Si bien los resultados publicados hasta ahora son indicativos de que la cirugía radical puede estar asociada con una supervivencia más larga en comparación con la quimioterapia sola, una tesis doctoral publicada en Madrid, en 2017, finalmente, reportó que de 474 pacientes con análisis de supervivencia, la mediana fue de ocho meses (16). Además, un pH bajo en el líquido pleural mostró el fracaso de la pleurodesis, debido a una mayor actividad tumoral en la cavidad pleural y a una mayor actividad metabólica que favorezca la reacumulación de líquido y, por tanto, una pleurodesis fallida. Resultados del estudio del líquido pleural como un pH mayor o igual a siete pueden presentar supervivencia, aunque sin significación estadística; así mismo una una glucosa mayor a $60 \mathrm{mg} / \mathrm{dL}$ se asoció 
con mayor supervivencia. Lo reportado en este caso demuestra que el uso de pleurodesis química incrementó la supervivencia del paciente en más de cuatro años.

Las opciones terapéuticas para esta enfermedad son pocas y el método para emplear un tratamiento es ampliamente controvertido, entre los que se incluyen procedimientos quirúrgicos, quimioterapia y radioterapia. Múltiples ensayos han concluido que la tasa de sobrevida varía en pacientes a quienes se les realice neumonectomía, que tiene mayor supervivencia comparada con aquellos a quienes únicamente se les realiza un procedimiento quirúrgico extrayendo pleura o pulmón. Esto se podría considerar como pilar fundamental del tratamiento $(17,18)$.

Se deben realizar estudios exhaustivos de antecedentes en los pacientes, pues la clínica de esta enfermedad es inespecífica y no permite la identificación oportuna. De ser así, se puede brindar tratamiento pertinente que ayude a mejorar la calidad de vida y el pronóstico de la enfermedad.

\section{Agradecimientos}

ـ os autores agradecen a la familia del paciente, su colaboración y aceptación de este caso.

\section{Contribución de los autores}

Tuanita Betancourt contribuyó con la escritura y búsqueda de información.

Juan Carlos Quintana y Juan Carlos Hernández: asesores de correcciones y organización del componente estructural.

Carlos Alberto Rodríguez: médico tratante y aportó a las correcciones.

Carlos Augusto Rojas: aportó con la lectura histopatológica de las muestras del paciente.

\section{Descargos de responsabilidad}

T os autores no aceptan responsabilidad por exclusión de datos o resultados de la aplicaUción del método indicado en este artículo. 


\section{Conflictos de intereses}

$\mathrm{N}^{\text {menurodocharata. }}$

\section{Referencias}

1. Bibby AC, Tsim S, Kanellakis N, Ball H, Talbot DC, Blyth KG. Malignant pleural mesothelioma: an update on investigation, diagnosis and treatment. Eur Respir Rev. 2016;25(142):472-86. https://doi.org/10.1183/16000617.0063-2016

2. Price B. Analysis of current trends in United States mesothelioma incidence. Am J Epidemiol. 1997;145(3):211-8. https://doi.org/10.1093/oxfordjournals.aje.a009093

3. Abeloff MD, Armitage JO, Niederhuber JE, Kastan MB, McKenna WG. Abeloff's clinical oncology. Philadelphia: Elsevier Health Sciences; 2008. https://doi.org/10.1016/B978-14557-2865-7.00110-7

4. Wagner JC, Sleggs CA, Marchand P. Diffuse pleural mesothelioma and asbestos exposure in the North Western Cape Province. Br J Ind Med. 1960;17:260-71. https://doi.org/10.1136/ oem.17.4.260

5. Nicholson WJ, Perkel G, Selikoff IJ. Occupational exposure to asbestos: population at risk and projected mortality--1980-2030. Am J Ind Med. 1982;3(3):259-311. https://doi. org/10.1002/ajim.4700030305

6. McDonald JC. Health implications of environmental exposure to asbestos. Environ Health Perspect. 1985;62:319-28. https://doi.org/10.1289/ehp.8562319

7. Spirtas R, Beebe GW, Connelly RR, Wright WE, Peters JM, Sherwin RP. Recent trends in mesothelioma incidence in the United States. Am J Ind Med. 1986;9(5):397-407. https:// doi.org/10.1002/ajim.4700090502

8. Aisner J. Current Approach to malignant mesothelioma of the pleura. Chest. 1995;107(6, Suppl):332S-44S. https://doi.org/10.1378/chest.107.6_supplement.332s

9. Arnold DT, Maskell NA. Biomarkers in mesothelioma. Ann Clin Biochem. 2018;55(1):4958. https://doi.org/10.1177/0004563217741145

10. Oropesa C. A, Soto G. S. Derrame pleural neoplásico. Cuad Cir. 2002;16(1):92-9. https:// doi.org/10.4206/cuad.cir.2002.v16n1-15

11. García Atienza EM, Val Jiménez CL, Navarro Ruiz S, López-Torres Hidalgo J. Mesotelioma pleural maligno. Medicina Paliativa. 2017;24(1):47-50. https://doi.org/10.1016/j.medipa.2014.11.001

12. Al-Alao BS, Parissis H, Rychlik IJ, Graham A, McGuigan J. Prognostic factors in malignant pleural mesothelioma: role of talc pleurodesis. Asian Cardiovasc Thorac Ann. 2012;20(4):443-9. https://doi.org/10.1177/0218492312449633

13. Bielsa S, Panadés MJ, Egido R, Rue M, Salud A, Matías-Guiu X, et al. Rentabilidad del estudio citológico del líquido pleural en el derrame maligno. An Med Interna. 2008;25:173-7. 
14. Rodríguez Panadero F. Diagnosis and treatment of malignant pleural mesothelioma. Arch Bronconeumol. 2015;51(4):177-84. https://doi.org/10.1016/j.arbres.2014.06.005

15. Weder W, Stahel RA, Bernhard J, Bodis S, Vogt P, Ballabeni P, et al. Multicenter trial of neo-adjuvant chemotherapy followed by extrapleural pneumonectomy in malignant pleural mesothelioma. Ann Oncol. 2007;18(7):1196-202. https://doi.org/10.1093/annonc/ mdm093

16. Díaz RM. Parámetros asociados a la eficacia de la pleurodesis y a la supervivencia en pacientes con derrame pleural maligno [tesis doctoral]. Madrid: Universidad Complutense de Madrid; 2015.

17. Moreno Balsalobre R. Tratamiento quirúrgico del mesotelioma: Sí [Internet]. Revistadepatologiarespiratoria.org. https://www.revistadepatologiarespiratoria.org/ descargas/pr_13-s1_30-32.pdf

18. Abdel-Rahman O, Elsayed Z, Mohamed H, Eltobgy M. Radical multimodality therapy for malignant pleural mesothelioma. Cochrane Database Syst Rev. 2018;1(1):CD012605. 